\title{
Face Perception Methodologies with Principal Component Methodology Algorithm
}

\author{
Syed Umar \\ Department of Computer Science Engineering \\ MLRIT, Hyderabad \\ P. Gayathri \\ Department of Information Technology \\ GRIET, Hyderabad
}

\begin{abstract}
In this article, two new facial recognition techniques based on a single vector Decomposition (SVD). Here is the method of the principal component method (PCM) and linear vector discriminate (LVD) applied to the facial features, the main component of the detection face recognition problem. The human face is full of information, but it works all the information that is time-consuming and inefficient. Better, special and important information and other information that is not useful that the system is an effective solution. The main component of the method aspects of face recognition. Vectors and face their own value calculated by a series of portraits. New faces projected onto the surface of the expanded clean room and a weighted sum of their face. The weights are used to detect the face. The Euclidian distance in time and space for growth in the face of this complexity using two singular value decomposition methods. The simulation results show that the effectiveness of the proposed faces recognition technology. Here is the face used principal component method and linear discriminate method series of special value. To Test, the data shows that the face of the proposed algorithm influences the complexity its accuracy. Basis, it is concluded that the major part of the flex can achieve good results than other methods. The results also realized that the time complexity of the proposed method significantly reduces promote the effective implementation of the proposed method.
\end{abstract}

Keywords: Principal Component Method, SVD, LVD

Received: 29 April 2019, Revised 3 August 2019, Accepted 14 August 2019

DOI: $10.6025 / \mathrm{jmpt} / 2019 / 10 / 4 / 133-137$

(C) 2019 DLINE. All Rights Reserved

\section{Introduction}

Problem with face recognition project has been much studied in recent years. This problem has been studied in the field in different ways. This is because many of the other benefits of biometric face plate method [1] [2]. Most volunteer work biometric user to identify and hands, standing in front of the retina or Iris recognition camera in a fixed position on the other side, and the fingerprints hand geometry. However, their looks can only be achieved; no course work or participating in the film is to buy the part of the room. This is particularly useful for the purposes of security and surveillance. Many other face recognition application in daily life, as a human being to recognize the card, passport, criminal, sources etc. [3] changes in the appearance of the image can be divided into two groups: internal and external reasons factors. A) With in the characteristic features of the face and the only independent thinkers. These factors can be divided into two groups: internal and relationships with employees. The internal

Journal of Multimedia Processing and Technologies Volume 10 Number 4 December 2019 
factors are responsible for looking at different types of the same year some people, for example, the shape and form (form of hair, glasses, cosmetics, etc.).

However, people are responsible for different aspects of the human variety of factors, ethnic and male and female are a few examples. B) External factors, changes in the way favored by the interplay of light and facing the viewer. These factors include lighting, modeling, coverage and ultrasound parameters (such as resolution, focus, image, sound, etc.). PBS [4] identity-based approach to the famous and linear perspective, which takes into account all the parts of the face, how to enter data into a form recognition system. Under the supervision of teachers in each category, brand or price model in the model are known. Furthermore, the maintenance of one or more of the system creates a group the transfer of the natural order; it means the end of the model is not yet known. The method is based on the PCA Loev converted Karhunen (klt) [5] [6].

Given the purpose of establishing a social relationship, it is important to convey identity and feelings. If we gather intelligence and physical appearance is suspicious, the human ability to recognize faces. You can identify thousands of beautiful in their lives and learn to recognize, first, after many years of separation. This ability is very strong, despite the major changes in the image, rabbits, viewing conditions, comments, age and concerns, such as glasses, a beard or changes the way hair. Mug shot sign music programs, consolidation of credit card as a driver and pilot, limited to the gate and the control group in all areas of conduct on the implementation of a viable pattern recognition system. Accreditation form has become a major problem for many applications such as security systems, credit card verification and identification of crime [7] - [10].

\section{Study of Literature}

Martinez.[7] As image recognition algorithm as Hilda (Linear discriminate analysis) the proposal is better than that based on PCA (method main component). A full list can be better to have a small layer of the PCA and PCA sensitive Hilda various training camps.

In Xiaofei they explained clearly various methodologies called Laplace complexion. Using multiple security plan (MSP), next to the beautiful scenery subspace research. Unlike the principal component method (PCA) and Linear discriminate method (LDM), who see only the Euclidean structure of the individual, to find a new place in PGD, The transition from the light, and seeks to eliminate or reduce.[8]

In Liu .If you set the size of the necessary quality to make an act of discrimination against the main system flow pattern recognition (FPR). Not surprisingly, De, features such as model-based testing (PCA) and Linear discriminate method (LDM), and provide real and reliable solutions to the problems in FR mask change. Writer found that the real machines based on the discriminate method, De method fairer distribution system. [9]

According to Lou. Here determine the Linear discriminate method (LDM), according to a lesser extent, Optimization measures is not directly related to skills indexing feature presentation. Top of the authors of the new algorithm financial crime or money and quality. A method was compared in terms of classification, the FPR frequently used data on the plane. The results show that the idea of working often better than traditional FR, as Eigen Next, Fisher met and Dr.Alda. [10]

\section{Face Recognition Methodologies}

\section{1. Linear Discriminate Method (LDM)}

LDA The goal is to find a good and interesting way to represent the face of a vector space. But to use the information in the class is useful in the identification of the projects could be; Linear discriminate Method (LDM) to obtain the vector with the lower space that a clearer distinction between classes. LDA seeks the optimal transformation that seeks to maximize the amount of

$$
\text { 1. } J_{L D A}(W)=\arg \max W\left|W^{T} S_{B} W\right| \backslash\left|W^{T} S_{B} W\right|
$$

SB is a matrix, in which the class and the class scatter matrix, SWD. Thus, the general problem of Eigen values can be found, for example, the projection of vector W SW SB -1 eigenvectors of the largest eigenvalues. If the sample size is smaller than the size of the sample, on the other hand, the SW unit and cannot be calculated directly from SW-1 SB. There are two main problems of the linear discriminate method (LDM) face. First, the LDA is not stable due to the small size of the training sample. Another is that

$134 \quad$ Journal of Multimedia Processing and Technologies Volume 10 Number 4 December 2019 
it closes the distribution of samples of different data cluster of the class multimodal

\subsection{Principal Component Methodology [PCM]}

S-dimensional vector representation of each page within the educational picture, principal component method (PCM) is often found dimensional subspace vector in the direction of maximum variation within the original image. New $\mathrm{T}<<$ subspace dimension, usually less. If these factors are treated as random variables PCA basis vectors is defined as the eigenvector matrix suspension. Eigen Face PCA algorithm to reduce the size of the search vectors that best account for the distribution of the images in the entire image area. This vector defines the look and image of the subspace is called the face. All types of training sets are scheduled before bed, to find a set of weights represents the contribution of each vector in the open space. Image recognition test, it takes a special test chamber the image to get the desired weight set. Compared with Euclidean distance between the test images in the face of heavy weight training set, the faces of the photo test can be set [11-13]

\section{Face Perception Methodologies \& Examing}

1. Select a training set of images. Let's shaky legs $I(x, y)$, the size of $N 2(N \times N)$, which is an $\mathrm{N}$-dimensional column vector $2 \mathrm{x} 1$ set of images that can be mapped to a point in the data-M "magazine" high-dimensional I1, I2 ...... IM.

2. Calculating the average performance kit and a set of normalization by removing the worst of all in the training set.

3. Pick your vector corresponding to the maximum flow Eigen values in order to have an idea or less expensive passenger projections.

4. But think of all the sides, and the bottom of the face. Each of centralized training OI projected images Eigen mode. If you want to project the image of the picture Eigen mode, calculate the dot product of vectors sorted.

$$
\begin{aligned}
& A=1 / M \sum_{n=1}^{M} 1_{n} \\
& \Phi_{i}=I_{i}-A \\
& C=1 / M \sum_{n=1}^{M} \Phi_{i} \Phi_{n}^{T}=X X^{T} \\
& X=\left[\Phi 1, \Phi 2 \ldots \Phi_{M}\right]
\end{aligned}
$$

\section{Experimental Analysis and Simulation of Various Images}

In this section we are clearly explains and simulates how the image or face perception can be done using various techniques like PCM and SVD with LDA and EVD these are shown in the below

\subsection{Face Perception using SVD-PCM}

In this Simulation we are combining SVD with the PCA for the Face perception analysis, so for the implementation of PCA w.r.t SVD methods. The below figure shows the exact analysis of how the SVD and the PCM will be used for the recognition of faces and to reconstruct it exactly.

\subsection{Face Perception using SVD - LDA}

In this analogy we are using the SVD technique with LDA algorithm to know exact analysis of the face. In this methodology an inserted image is identified and check with the real image whether it is more accurate and exact to the original image and it begins the test analysis also when we compared with SVD PCA with the SVD-LVD is the best methodology which Will give better results.

\section{Conclusion}

In this paper we are presenting about the Dimensional reduction and to interface for face perception. It is widely used in many cases where the high-dimensional data, such as face recognition. In this article, to reduce the face detection information of the industry, then using the singular value decomposition (SVD) for the projection matrix, large appear in three space dimensions 


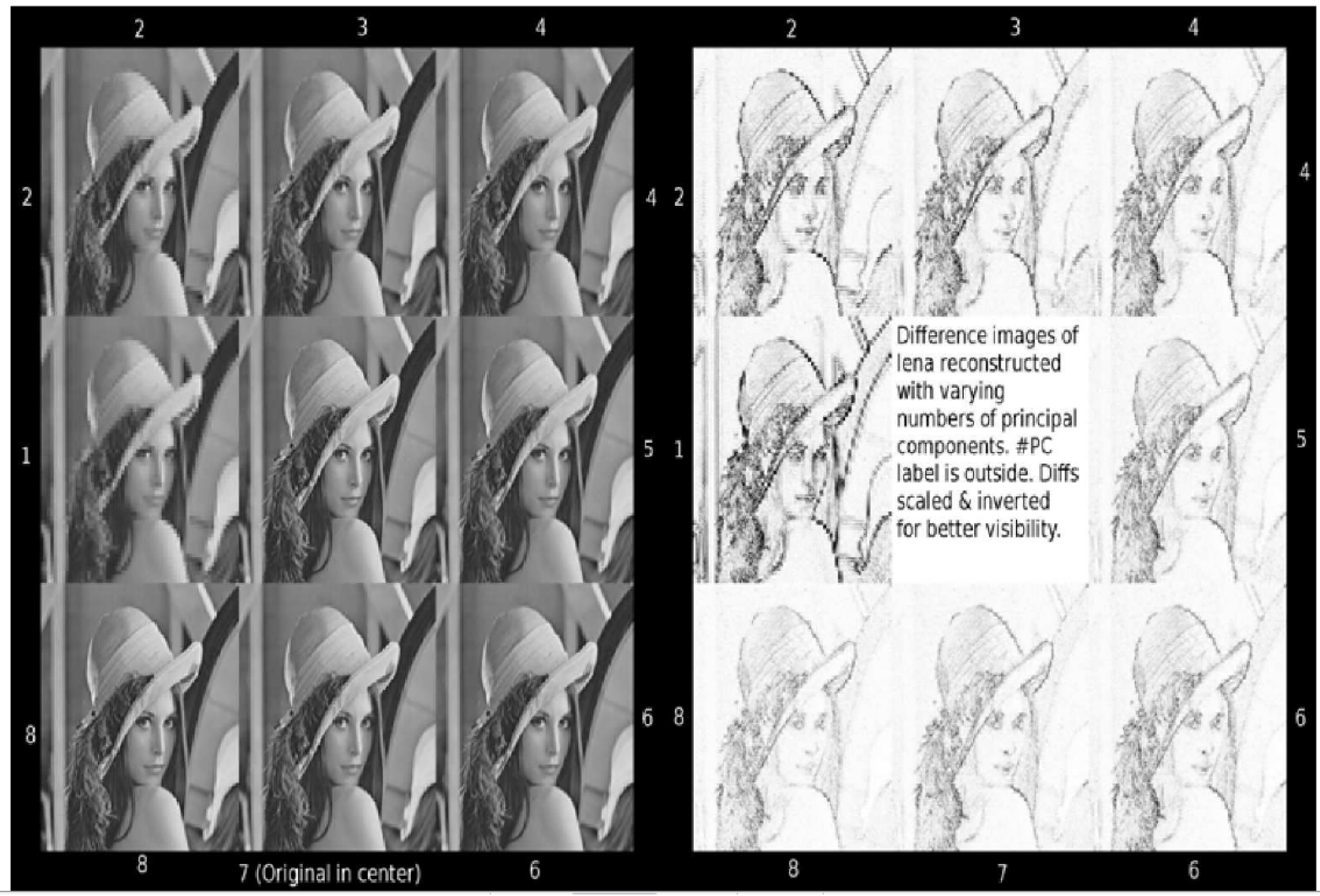

Figure 1. Face Perception using SVD-PCM

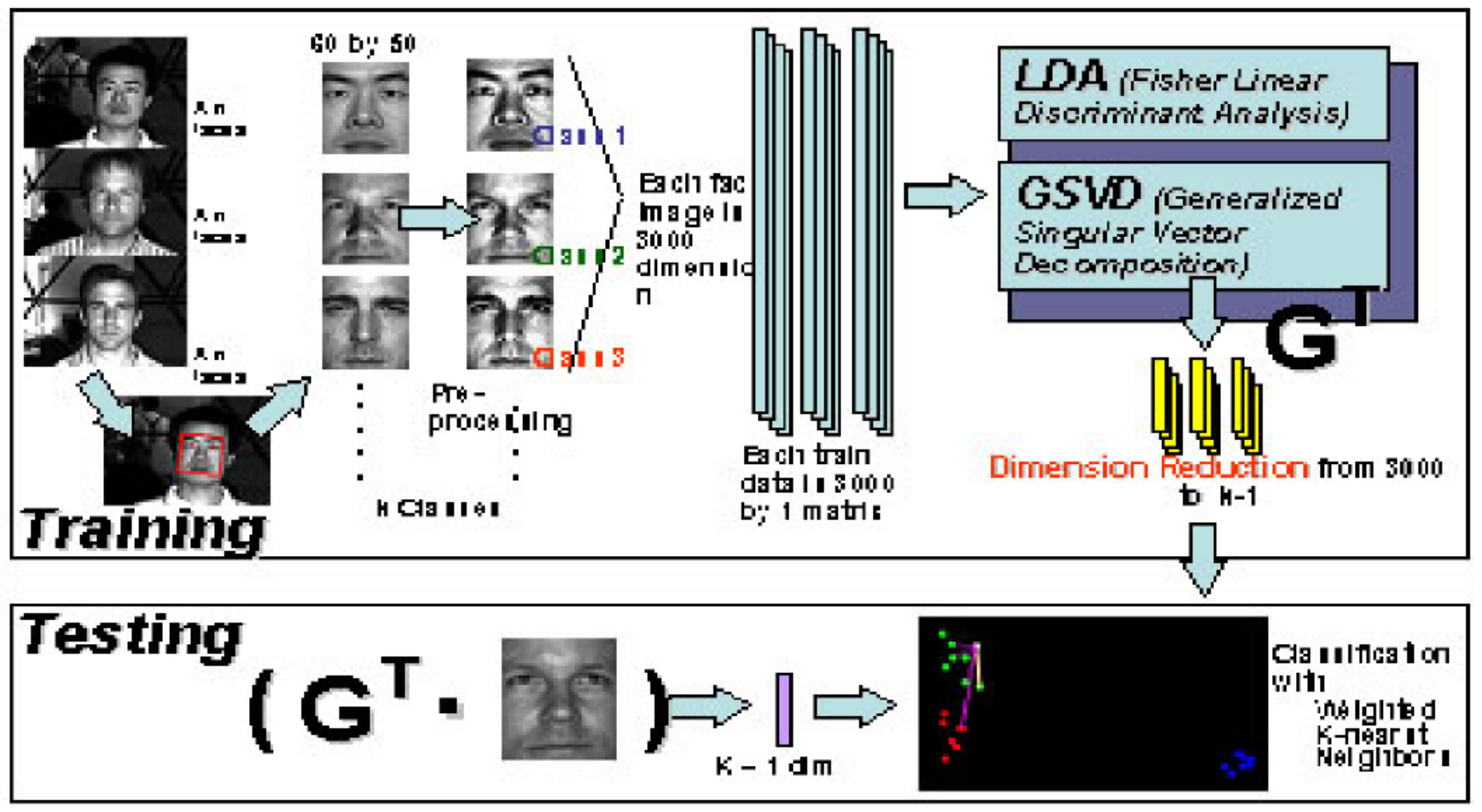

Figure 2. Face perception using SVD-LDA 
poor image. Experience shows the performance of the algorithm proposed in our database, and the results were compared with SPS Eigen value decomposition (EVD) indicates that the proposed themselves in the system a little better than methods previous how to reduce the time to give complexity without affecting its accuracy. So by using the EVD and the SVD we are getting more accurate and good resolution images.

\section{References}

[1] Babies, R. Hamid Jafar or Arabic. (2009). A l'enquête de en face recognition technology, Journal of information processing systems, Vol.5 June 2, 2009.

[2] Comme. Tolba, A.H. El-Baz or A.S. El Harby. (2006).“visages Survey December: A Revue de Littérature”, Revue Internationale des or information INGENIERIE, in February 2006.

[3] Han, CC. (1997). Hy Mark Liao, G. 1. Yu Chen LH or. Quick pre-TRAITEMENT basic structure detecter. Au TRAITEMENT Image Analysis, p. 469 to -476. Springer, 1997.

[4] AP Matthew Turk à Alex Pentland. (2004). "Face Recognition Using Eigen Face, In: Proc. IEEE Computer Vision Conference or SA Pattern Recognition, p. 586-591, 1991. I Tamal Bose logo NUMERIQUE à images, Asie: Wiley, 2004.

[5] Moghaddam, AA. Pentland. (2002). Visuelle Probabiliste learn some opposition representation. method of recording Artificielle infor, B. mation, IEEE Cash SA, 19 (7) 696 to 710.2002.

[6] Hsuan Yang Ming., David J Kreigman Narender Ahuja. (2002). "face detection in images: a survey of money Motif de l'Information methodArtificielle IEEE Vol 24, No. January 1, 2002.

[7] Zhao Haitao., Chi Yuen Pong. (2008). "Incremental Linear discriminant method Face Recognition, IEEE Transactions on Systems, Man, or Cybernetics Part B: Cybernetics, Vol 38, Issue 1, Love: 210-221, 2008.

[8] SH Leng., Hym Liao., Han CC., MON Chern. (1998). à YT Liu. With face detection feature Modele facial geometry, a good strategy. Recognition recording, 31 (3) 273-282, 1998.

[9] Sirovich à M. Kirby. (1987). Low-parity approach dimensionnelle to describe the man, J. Opt. Soc. Un m. A, Vol. 4, No. 3, March 1987,519-524.

[10] PN Belhumeur., JP Hespanha DJ Kriegman. (2002). Eigen Face vs. Face Fisher: Survey using special MINISTER LINE-class projection. method of recording Artificielle à l'information, IEEE Cash SA, 19 (7) 711 to 720.2002.

[11] LF Chen., Hym Liao., MT I, 1. C. Lin, et G. 1. Yu. (2000). The new identification system based LDA face minor problems can be solved Taille de l'échantillon. Recognition Motif, 33 (10) 1713 to 1726. 2000.

[12] Pentland, A., Turk, M. (1991). Eigen equivalent Face ID. Journal of Cognitive Neuroscience, 3 (1) 71-86, 1991.

[13] Hu, H., Zhang, P., From the tower, F. (2010). "face recognition to improve the discriminant method MINISTER LINE", IET Computer Vision, Vol 4, No. 3: 195-208, 2010.

[14] Bansal, A., Mehta, K., Arora, S. (2012). "Face Recognition Using PCA for writing algorithms of LDA", Internationale Conference on Advanced Computing and Communication south (Hb), 2012 Page (s): 251-254, 2012. 